\title{
La influencia del contexto como práctica comunicacional: el desafío de la integración en la sociedad de la información
}

\author{
Santiago Aragón \\ Universidad Nacional de Lomas de Zamora. Doctorando en Universidad Austral. \\ sansanaragon@gmail.com
}

Fecha de finalización: 31 de agosto de 2020 .

Recibido: 31 de agosto de 2020

Aceptado: 19 de octubre de 2020.

DOI: https://doi.org/10.26422/aucom.2020.0902.ara

\section{Resumen}

El artículo propone explorar la importancia de la comunicación y de los sistemas de información para la articulación entre las relaciones globales y las prácticas territoriales. El formato de ensayo permite indagar en las tensiones y las dinámicas de colaboración que resultan de la coexistencia entre la institucionalidad local y la sociedad red.

Las condiciones sobre las que se construye la conversación pública se modifican en un escenario en el que la producción y la circulación de información ocupan el centro de la escena. Existen nuevos mecanismos que obligan a revisar la forma en la que las instituciones políticas y las organizaciones mediáticas gestionan sus canales de expresión y discusión.

Se pretende dilucidar dos desafíos: ¿pueden los sistemas políticos sustituir una dinámica de representación por otra de negociación sin ver alterada su función y jerarquía? ¿Los territorios logran inscribir sus prácticas particulares en el registro que propone la sociedad red?

La distinción entre una espacialidad capaz de sobrevivir en la convivencia con la sociedad de la información y otra que queda excluida permite reflexionar sobre las condiciones de adaptación. El interrogante es en qué medida las posibilidades de emerger de las identidades locales están relacionadas con su capacidad de expresarse y vincularse en el marco de una práctica comunicacional que asocie la influencia de lo global con la vigencia de lo cultural.

Lo potencialidad de convertir lo periférico en contexto, integrando lo local a través de dinámicas colaborativas, surge como un campo común que integra, en su análisis, la cultura y la comunicación.

Palabras clave: sociedad red, sistemas globales, espacio, territorio, cultura, contexto, comunicación. 


\title{
How context influences the practice of communication: the challenge of integration in our information society
}

\begin{abstract}
This article explores the importance of communication and information systems in the articulation of global relations with territorial practices. We adopt an essayistic format, which allows us to probe the tensions and collaborative dynamics that emerge from the coexistence between local institutions and connected society.

The conditions under which the public conversation unfolds are modified in a context where the production and spread of information occupy center stage. There are new mechanisms in place that force us to rethink how political institutions and media organizations manage their channels for expression and discussion.

In what follows, we seek to answer two different questions. First, can political systems substitute a representational dynamic for another one centered on negotiation, doing so without altering their function and importance? And second, can territories embed their practices within the record of connected society?

There is an important distinction to be made between spaces that can survive and coexist within our information society and those that, instead, are excluded from it. This distinction allows us to reflect on the conditions of adaptation. The question is to what degree does the possibility of emergence, for local identities, depend on their capacity to express themselves and network in the context of a communicational practice that can associate global influence with the continued relevance of culture.
\end{abstract}

The potential to transform the periphery into context, integrating the local through collaborative dynamics, emerges as a field that combines, in its analysis, both culture and communication.

Keywords: connected society, global systems, space, territory, culture, context, communication.

\section{A influência do contexto como prática de comunicação: 0 desafio da integração na sociedade da informação}

\section{Resumo}

O artigo se propõe a explorar a importância dos sistemas de comunicação e informação para a articulação entre relações globais e práticas territoriais. $\mathrm{O}$ formato de ensaio permite investigar as tensões e as dinâmicas de colaboração que resultam da convivência entre o quadro institucional local e a sociedade em rede.

As condições sobre as quais se constrói a conversa pública são modificadas em um cenário em que a produção e a circulação da informação ocupam o centro da cena. Existem novos mecanismos que exigem uma revisão da maneira na qual as instituições políticas e as organizações de mídia gerenciam seus canais de expressão e discussão.

Pretende-se elucidar dois desafios: os sistemas políticos podem substituir uma dinâmica de representação por outra de negociação, sem ver alterada a sua função e hierarquia? Os territórios podem registrar suas práticas particulares no registro proposto pela sociedade em rede?

A distinção entre uma espacialidade capaz de sobreviver na convivência com a sociedade da informação e outra que é excluída permite refletir sobre as condições de adaptação. A questão é em que medida as possibilidades de emergentes identidades locais estão relacionadas à sua capacidade de se expressar e se vincular, no quadro de uma prática comunicacional que vincula a influência do global à validade do cultural. 


\begin{abstract}
A potencialidade de contextualizar o periférico, integrando o local através de dinâmicas colaborativas, surge como um campo comum que integra, na sua análise, a cultura e a comunicação.

Palavras chave: sociedade em rede, sistemas globais, espaços, cultura, contexto, território, comunicação.
\end{abstract}

\title{
Sistemas globales y prácticas culturales
}

El artículo pretende explorar los escenarios de convergencias y tensiones entre la dimensión particular de la cultura y la perspectiva universalista de la sociedad red. Se abordan los desafíos de la convivencia entre sistemas en el escenario global, de qué manera esta dimensión se articula con lo local y cómo la comunicación puede generar los mecanismos para representar las particularidades del contexto y lograr influencia en el espacio público.

El surgimiento de un modelo espacial, de configuración transnacional, dinámica sistémica y base informacional reconfigura el alcance institucional y provoca tensiones entre la dimensión universal y la local.

Desde la perspectiva de la antropología simbólica (Geertz, 2005), la cultura puede ser entendida como un mecanismo basado en conductas que adquieren un alto grado de particularidad, ya que estudian las relaciones sistemáticas que se dan en un lugar y un momento determinados. La coexistencia entre distintos valores y formas de interpretar la realidad, sus usos y modos le otorgan relevancia a la variable territorial, considerada, además, desde su perspectiva histórica.

Esta distinción entre una espacialidad que se adapta a la sociedad de la información y otra que no logra integrar sus prácticas en lo universal expresa una idea de inclusión y exclusión en torno a la territorialidad, que se extiende a los sistemas que gestionan el entorno local.

¿Puede lo político-institucional sustituir una dinámica de representación e imposición por otra de negociación y delegación sin ver alteradas sus funciones y su lugar en el ordenamiento social? ¿Está en condiciones de incorporar nuevas prácticas sobre las cuales se sustente la construcción de comunidad, adquiriendo, desde lo procedimental, la capacidad de accionar en red en sustitución de un poder ejercido en forma estructural?

La coexistencia de prácticas particulares, desarrolladas en el marco de cada territorio, con un modelo universal con base informacional genera una tensión entre una concepción cosmopolita, de institucionalidad y cultura globales, y una multipolar, en la que las características de cada territorio trasladan la diversidad -y sus desafíos-, al escenario extendido (Mouffe, 2007).

La necesidad de articulación entre los valores basados en la circulación y el acceso -en la lógica transnacional-y los de identidad y pertenencia -que quedan reservados 
al ámbito local- explora dinámicas de colaboración que garanticen la coexistencia. En otros casos, las dinámicas de colaboración se ven imposibilitada por las características de los valores que se sustentan en el territorio. En estos escenarios, el análisis se concentra en las tensiones que se originan entra la propuesta global y las perspectivas regionales.

En el planteo de Clifford Geertz (2005), "'la cultura es pública porque su significación lo es" (p. 26). Desde esta perspectiva, la cultura aparece como un producto en el cual la interpretación juega un rol relevante en una dinámica de emisión y reconocimiento. La identidad se construye en un proceso de compresión de carácter colectivo. No se completa hasta que una producción no logra inscribirse y ser reconocida en el ámbito de lo social.

La comunidad, los lazos territoriales, la comunión sobre problemáticas comunes y los hitos que conforman la historia desarrollan un campo de sentido en el cual determinados signos cobran valor y se tornan vigentes, o no, y son reconocidos o desaparecen. Este alto grado de particularidad expresado por la idea de cultura aparece como una alternativa a los sistemas globales. En esta perspectiva, lo territorial adquiere un valor específico.

En lo metodológico, esta interpretación se justifica en lo actuado frente o lo supuesto. "lo real" adquiere vigencia en un lugar y un momento determinados. El contexto es determinante en el sistema. La etnografía se concentra en los rasgos particulares que adquieren relevancia. Así, se inscribe en el discurso social; su tarea "es interpretativa, lo que interpreta es el discurso social y la interpretación consiste en tratar de rescatar 'lo dicho"' (Geertz, 2005, p. 32).

El registro de "lo que es" enfrenta las abstracciones del sistema y su pretensión de universalidad. Los sujetos expresados como generalidad ${ }^{2}$ no constituyen una categoría de análisis. Solo puede interpretarse lo actuado y sistematizarse lo real.

Otras corrientes también relativizan la idea de una cultura universal y su influencia en la convivencia con realidades locales. El neoevolucionismo sostiene que "las culturas son consideradas como entidades discretas situadas en diversos nichos ecológicos, que van adquiriendo formas diversas a través de la adaptación a diversas condiciones" (Sztompka, 2012, p. 141). La capacidad de observar relaciones sistemáticas entre elementos de naturaleza diferente aporta la necesidad de hablar de "las culturas", considerando la complejidad como una puesta en valor.

Clifford Geertz es considerado el padre de la antropología simbólica. La perspectiva semiótica incorporada al estudio de la cultura otorga valor a la interpretación de las relaciones sistemáticas que se dan en un contexto determinado. La "descripción densa" como variante de la etnografía se convierte en la base metodológica sobre la cual se organiza la observación.

2 Geertz hace referencia a la imposibilidad de analizar al Hombre, con mayúsculas, ya que esta es una construcción ideal sin base real. Su idea de "hombre con minúscula" hace referencia a un objeto que solo puede ser abordado desde una perspectiva que incorpore un alto grado de particularidad capaz de contener la diversidad de la especie. 
El grado de particularidad se extiende a la reflexión sobre el sujeto. La caracterización basada en rasgos comunes, base de un sistema único de identidad y funciones, se enfrenta con la complejidad de factores que intervienen en la configuración del sujeto. Estos se orientan a las relaciones que establece con el territorio y sus semejantes en el marco de problemáticas y de influencias específicas.

Geertz (2005), sobre el presupuesto de la universalidad, afirma:

\begin{abstract}
no se pueden hacer generalizaciones sobre el hombre como hombre, salvo que este es un animal sumamente variado, o que el estudio de la cultura en nada contribuye a revelar tales generalizaciones. Lo que quiero decir es que ellas no habrán de descubrirse mediante la búsqueda baconiana de universos culturales, una especia de escrutinio de la opinión pública de los pueblos del mundo en busca de un consensus gentium que en realidad no existe (p. 48).
\end{abstract}

La apuesta por la universalidad representa una imposición que no es exclusiva del paradigma de la globalización. Todo sistema político con ambición hegemónica ha intentado universalizar sus prácticas para acompañar, desde la cultura, la pretensión de totalidad reclamada por el sistema. La historia ofrece ejemplos en los que la hegemonía política reclama un modelo cultural que acompañe el proceso.

El individuo, esa particularidad moldeada por lo social, adquiere, bajo esta ambición de hegemonía, un carácter político. El alineamiento de lo político con lo institucional prescinde de la variable territorial en la consolidación de la modernidad. De tal grado es la simbiosis entre civilización (universalidad) y cultura que, en gran parte de los siglos XIX y XX, es dificultoso encontrar referencias específicas de la idea de cultura que no estén inscriptas en una perspectiva supranacional (Eagleton, 2001). Esta universalidad, con base institucional, se incorpora al terreno de lo económico, de lo social y de lo cultural.

Las diferenciaciones se disuelven en el tránsito a establecer condiciones de ciudadanía. Distintas culturas empiezan a compartir, en torno a la idea de civilización, ambiciones relativas a sus formas de organización política, social y económica. La dificultad de conciliar estos sistemas con la diversidad de los territorios a los que pretende aplicarse se expresa en distintos grados de desarrollo social. Munis Sodré (1998) explica que "el individuo ciudadano resultante es un hecho ideológico. De él parten las ciencias sociales y humanas para la formulación del objeto teórico llamado 'sociedad moderna"' (p. 47).

La imposibilidad de articular la cultura y lo global como parte de un mismo concepto surge de la confrontación entre la ambición de universalidad de sujetos y sistemas con el alto grado de particularidad expresado en sus relaciones. 
La importancia que adquieren los sistemas de información, expresados en la idea de sociedad red, ${ }^{3}$ y la complejidad de las variables que explican lo territorial producen la necesidad de un doble ejercicio destinado a reconocer, como parte del mismo análisis, la influencia de lo global y la vigencia de la cultura.

Definir los alcances de una "cultura global" no implica desconocer la importancia de las variables universales sobre lo regional y lo local y el alto grado de influencia en instituciones y sujetos. El interrogante es qué forma adquiere la estructura de lo universal para relacionarse con lo particular y en qué medida esto se expresa en dinámicas de colaboración o en tensiones.

La cultura como concepto pone en entredicho el vínculo, sugerido como natural, entre el sujeto y los sistemas universales y, como consecuencia, se convierte en un antídoto ante la prevalencia del individualismo. Esto determina el alto grado de influencia del concepto de "cultura" en el concepto de "sujeto", ya que sin esta relación no podrían comprenderse las características del sujeto, pues "no existe una naturaleza humana independiente de la cultura" (Geertz, 2005, p. 55).

\section{La información como sistema}

La reflexión sobre las posibilidades de articulación entre la sociedad red y las territorialidades locales involucra el rol de la información en la construcción de identidad, en la dinámica de las instituciones y en los modelos de vinculación entre sujetos. Este análisis comprende dos etapas: la consideración de cómo las condiciones de circulación imponen un mecanismo de acceso y uso -que ubica a la información en el centro del sistema, y a la conectividad como motor de un nuevo modelo de organización social- $\mathrm{y}$, además, cómo este articula su funcionamiento con la configuración territorial clásica, expresada por el Estado-nación.

\section{Nuevas condiciones de circulación}

El análisis de los sistemas globales y las prácticas culturales permite comprender el grado de influencia y relación que estas variables tienen en los conceptos de "sujeto", "instituciones" y "territorio". Para abordar estos cruces, es necesario ampliar la reflexión hacia la naturaleza de otros elementos que se involucran. Jonathan Friedman (2001) especifica

En los sistemas globales el proceso cultural no puede ser entendido si no se consideran los fenómenos de la hegemonía, de las identidades contrapuestas y los discursos dominantes y subalternos. Nuestro punto de partida, en términos globales, debe ser el centro mismo, puesto que sus propiedades culturales surgen en la expansión geográfica del sistema (p. 50).

\footnotetext{
Si bien el concepto forma parte de la obra de numerosos autores, es en la producción de Manuel Castells (2009) que la idea se desarrolla en forma más exhaustiva, analizando las implicancias de la información en las relaciones sociales, políticas y económicas. Por eso, la referencia de sociedad red, se orienta a la propuesta de este autor.
} 
En la negociación entre los sistemas globales y los procesos culturales, existen tensiones en el terreno de las identidades y de los discursos, que pueden ser pensadas en la lógica de centro y periferia. Lo que se percibe como universal es, en realidad, la expresión de un centro expandido que, en virtud de la eficacia del sistema, logra extender sus características más allá de su posición inicial.

El éxito de esta expansión hace que se presente como cultura global un conjunto de prácticas definidas sobre un imaginario de fronteras disueltas y sujetos en circulación, que desafían la supuesta obsolescencia de los límites territoriales e institucionales. Esta caracterización tiene impacto en los consumos culturales y en las formas de producción y de relación. La resultante es un modelo de relación basado en la libre circulación y el intercambio de información.

El tránsito del aquí y del ahora (Augé, 2008) prescinde del devenir histórico y acumula el tiempo con una lógica de microacontecimiento. Cada segundo parece desprendido del anterior y del siguiente. Cada vivencia se explica en sí misma, sin recurrir a prácticas colectivas ni coyunturas. Un presente extendido, un instante fractal que omite el devenir.

David Harvey (2004) define a este presente desbordado como "la reducción de la experiencia a una serie de presentes puros y desvinculados" (p. 72). Esto implica una materialización de lo inmediato y una jerarquización de lo vivido. Las prácticas relacionales que se dan en torno a estos sistemas tienen influencia en las sociedades de consumo, creando nuevos mecanismos de inclusión y de exclusión vinculados con la conectividad ${ }^{4} \mathrm{y}$ el acceso a la información.

Un desborde de la idea de tiempo, espacio y sujeto representado por la supresión del sentido histórico, en nombre de la instantaneidad, deriva en la idea de sobremodernidad. Las dificultades para imponer sentido, más allá de la inespecificidad de los derechos de acceso y circulación con base en la información, es evidente: sin experiencia histórica no hay cultura. Los sistemas globales evitan la reflexión acerca de su origen y destino, amparándose en "un marco sistémico global general en el que la globalidad no es una cuestión histórica sino estructural" (Friedman, 2001, p. 39).

La experiencia desprendida de su contexto histórico impone un tipo de circulación virtual que reivindica el derecho a la individualización, mediante la distinción, más allá de su colectivo original. La diferenciación entre sistemas globales y prácticas locales, con lo territorial como elemento distintivo, no puede ser abordada bajo la lógica de la confrontación entre la cultura de masas y la popular, sino a las diferencias entre lo universal y lo particular.

\footnotetext{
4 La sobremodernidad como concepto aparece desarrollado en Los no lugares: espacios del anonimato de Marc Augé (2008), así, se agrega a otras categorías que buscan referencia para explicar los términos de la salida de la modernidad, entre los que se cuentan la modernidad líquida, el capitalismo tardío y la posmodernidad.
} 
La articulación entre los conceptos de "posmodernidad" y de sobremodernidad se expresa en la condición de que para la aparición del segundo elemento es necesaria la imposición del primero. La posmodernidad es, más que un sistema de valores, la negación de todas aquellas premisas que organizaron la sociedad moderna (Dufour, 2007). Tras esta operación -de supresión de sentido- aparece la sobremodernidad, entendida como la imposición de lo instantáneo, lo universal y el imperio de las condiciones de circulación. La potencialidad de relacionamiento de los elementos, más allá de los límites territoriales, ratifica su condición de sistema.

La reflexión sobre los mecanismos por los cuales los sistemas globales construyen hegemonía plantea el desafío a los actores que forman parte del espacio público: las instituciones políticas y las organizaciones mediáticas deben incorporar nuevas formas de expresión y de discusión. Un escenario en el que la producción y circulación de información ocupa el centro de la escena, desbordando el rol tradicional reservado para dirigentes y comunicadores, obliga a revisar las prácticas sobre las que se desarrolla la conversación pública.

Hay, en la multiplicidad de expresiones que permiten los sistemas basados en la circulación de información, un desafío de carácter ético que se traslada a lo operativo. La premisa consiste en "aceptar la fragmentación, el pluralismo y la autenticidad de otras voces y otros mundos" (Harvey, 2004, p. 66). La función de la comunicación y el rol de los medios, en la construcción de poder, se vuelven centrales en este escenario.

Lyotard $(1997)^{5}$ menciona un cambio en el estatuto de la información y en las condiciones del saber. La información se transforma de bien de uso en bien de cambio, en virtud de un proceso de performatividad. El resultado de esta operación de traducibilidad ${ }^{6}$ es que los datos se convierten en una mercancía, privilegiando las condiciones de circulación y consumo.

El valor de la información en la constitución del sujeto no es exclusivo del abordaje posmoderno. La antropología simbólica ubica en esta relación la posibilidad de que un sujeto se constituya como tal. Esta caracterización divide a la información en genética y extragenética, considerando a la primera como la serie de instrucciones de funcionamiento con las que se cuenta biológicamente, y a la segunda, como los datos que se adquieren en la medida en que se establecen relaciones con el entorno. La información externa tiene un rol vital en la constitución del sujeto y un grado de particularidad expresado en los diferentes contextos en los que esta se produce.

\footnotetext{
Jean Francois Lyotard, en La condición postmoderna (1997), estructura una de las primeras obras cuyo contenido se dedica al advenimiento de esta nueva era. La originalidad del planteo se relaciona con ubicar a la posmodernidad como un cambio en las condiciones del saber y el uso de la información.

6 La idea de traducibilidad explica cómo la información pasa de lo analógico a lo digital, en el plano operativo, como expresión de lo que, a partir de la aplicación de la performatividad (jerarquización en función del uso) transforma a la información de bien de uso en bien de cambio.
} 
Esta afirmación tiene tres rasgos: la información es constitutiva, debe ser entendida como un bien de uso y es provista por la cultura. Clifford Geertz (2005) afirma que "entre lo que nuestro cuerpo nos dice y lo que tenemos que saber para funcionar hay un vacío que debemos llenar nosotros mismos, y lo llenamos con información (o desinformación) suministrada por nuestra cultura" (p. 55).

La información le agrega a su premisa de garantizar la adaptación individual a las prácticas colectivas la discusión acerca de cómo las condiciones de circulación redefinen su función y su alcance. En ese tránsito, deja de ser un instrumento para transformarse en el centro del sistema. De bien de uso a bien de cambio, se convierte en el insumo sobre el cual se constituye la sociedad red.

En este desplazamiento, lo que queda en el camino es el poder de la información de lograr inscribir lo individual en lo colectivo. En este nuevo marco, se puede operar dentro del sistema sin mediaciones sociales ni grupales y sin filiación a una territorialidad determinada. La información es el puente entre el sujeto y la red. Este aporte se sustenta desde una concepción técnica operativa, prescindiendo de categorías culturales. Harvey (2004) otorga este rol a las nuevas tecnologías, ya que "han eliminado la necesidad de asociar la producción masiva a la repetición masiva y han dado lugar a una producción masiva flexible de productos casi personalizados" (p. 95).

En nombre de la información empieza a imponerse una tendencia cierta a la individualización, expresada como una "dictadura del sí mismo"” (Contissa, 2000) amparada en consideraciones técnicas que privilegian el consumo y la circulación antes que el uso y la pertenencia colectiva.

El imperio de la información, su materialización como ideología del sistema y la defensa de las condiciones de circulación y acceso proponen categorías que pueden explicar cómo se funciona más allá de la red. El riesgo de esta exégesis se vincula con que "la información devora la comunicación y lo social, sin producir sentido verdaderamente" (Munis Sodré, 1998, p. 53).

Este fenómeno de "censura por asfixia" (Ramonet, 2003) justifica que la ausencia de sentido no está dada por la falta de información, sino por la imposibilidad de reflexionar sobre qué significan esos datos puestos en contexto. Cuál es la identidad que representan, a que práctica social refieren y, finalmente, a que ámbito territorial remit-

La idea de "dictadura del sí mismo" expresada en la obra de Tato Contissa ubica en la información, y su acceso, al cambio entre una comunidad basada en la "socialización disciplinaria", como expresión en la cual los elementos que forman el sistema comparten una mirada común respecto al sistema de valores que se desarrolla en una sociedad, y otra explicada por la "personalización" en la que el acceso a la información determina un tipo de sujetos autoconfigurables.

8 Ramonet (2003) define la "censura por asfixia" como el fenómeno por el cual el exceso en el consumo de información hace que se pierda la posibilidad de jerarquizarla, de contextualizarla y de considerarla bajo una perspectiva histórica. Es un efecto derivado de la circulación propuesta por la sociedad de la información, con efectos sobre los medios y la sociedad. 
Santiago Aragón

La influencia del contexto como práctica comunicacional: el desafío de la integración en la sociedad de la información

en son interrogantes que quedan inconclusos cuando la información es considerada un fin en sí mismo.

La imposibilidad de reemplazar lo que en términos locales representa la cultura, por lo que en términos globales define a un sistema, está dada por la improbabilidad de que esta sustitución arbitre los mecanismos de colectivización y comunidad que forman parte de las funciones originales. No obstante, existe un desplazamiento del sujeto entre sistema y prácticas, aun si las estructuras no son homologables.

Algunos interrogantes derivados de este desplazamiento entre lo global y lo local definen la naturaleza del escenario. ¿Existe una posible anomia de las instituciones que forman parte del Estado-nación? ¿Las culturas pueden arbitrar mecanismos de coexistencia para no perder sus funciones de relacionamiento? ¿En qué medida los sistemas globales están en condiciones de absorber las prácticas territorializadas de los sujetos que congrega?

\section{Los límites de la sociedad red}

La sociedad red ubica la capacidad de conectividad en el centro del proceso. Con independencia del conjunto, cada sujeto puede considerarse un nodo con funciones en una estructura relacional de carácter universal. El desafío ya no consiste en interpretar el todo a partir de las características de las partes, sino en "vincular los microsistemas al ecosistema general para entender la estructura normativa que subyace a la sociabilidad por plataformas" (Van Dijck, 2016, p. 280).

La cultura puede comprenderse bajo una perspectiva metonímica. Las características de sus prácticas hacen que las partes puedan expresar rasgos de lo colectivo. En esta dinámica de mayorías y representatividades, el estatuto de lo individual queda reservado a ofrecer elementos que permitan comprender el conjunto, ya que "lo que sean la mayoría de los actores, o los tipos especialmente influyentes de actores, modelará de forma decisiva la cualidad de la agencia" (Sztompka, 2012, p. 61).

La ausencia de referente dificulta las funciones institucionales. La dinámica de la conectividad reemplaza el ejercicio de las relaciones sistemáticas, ya que las condiciones de circulación se imponen a las de permanencia. El poder ya no se ejecuta dentro de un ámbito, se transforma en un medio de vinculación. En esta nueva lógica, no se confiere ni se delega, los sistemas representativos se tornan anómicos y, con ellos, la institucionalidad que los rige. La posibilidad de conectarse se transforma en el centro de la sociedad poniendo en valor una nueva característica, que define que "el poder no es un atributo sino una relación" (Castells, 2009, p. 34). En el camino inverso, la información se vuelve un concepto ordenador. 
La red incorpora la capacidad de articular lo individual con lo colectivo a través de un sistema de alcance global, "el etnólogo de las sociedades contemporáneas descubre, pues, la presencia individual en el universo globalizador" (Augé, 2008, p. 120).

Sin estadios de mediación entre lo individual y lo global, las prácticas culturales son postergadas por su ausencia de registro. Ni ellas ni las relaciones que representan desaparecen, solo pierden jerarquía en un sistema que valora la capacidad de unir elementos de características a través de funciones que los universalicen, sin perder la esencia de la individualidad.

La imposibilidad de concebir una cultura global -por la ausencia de prácticas colectivas territorializadas- no impide la vigencia de un sistema universal que transforma en identidad su capacidad relacional. Esta vinculación sustentable entre lo individual y lo global convive, en algunos casos, con lo que la cultura representa para la sociedad. La opción individual de privilegiar estos sistemas afronta el riesgo de la invisibilización de sus rasgos particulares y, con ellos, las prácticas de los sujetos representados.

Lo individual y lo universal se transforman así en dos elementos del mismo itinerario, sostenidos por la capacidad técnica de ser incluidos. La discusión acerca de los ámbitos en los que se desarrollan las estructuras que influencian al sujeto y los mecanismos de articulación de distintas expresiones espaciales permanecen. La determinación de las tensiones y dinámicas de cooperación se desplaza a la discusión entre lo global y lo local. Las características de este vínculo tienen consecuencias en los ámbitos institucional, cultural y político.

La determinación de cómo esta relación se materializa suele estar regida por dos tipos de "ficciones orientadoras", sustentadas por el deseo de invocar o negar un nuevo orden. Por un lado, la idea de que lo global desafía y desborda cualquier mecánica local, ya sea institucional o cultural, haciendo desaparecer toda expresión que no pueda ser reconocida desde un ámbito transnacional e incorporada a los consumos que la rigen. Por el otro, en una ficción de sentido inverso, que aquello que se invoca como global no es más que un conjunto de variables macroeconómicas amparadas en la idea de un capitalismo financiero global, que cuenta con expresiones de sentido que justifican estas prácticas, estableciendo una falsa dicotomía entre lo universal -presente extendido, futuro- y lo local como expresión del pasado. Las consecuencias se expresan en mecanismos de sustitución que afectan las premisas de la sociedad moderna por parte de las condiciones posmodernas expresadas en la sociedad red.

Estos dos enfoques, de signo opuesto, comparten la inespecificidad de sus postulados y desconocen la necesaria complementariedad entre los dinámicas globales y

\footnotetext{
La "ficción orientadora" aparece como concepto en la obra de Nicolas Shumway La invención de la Argentina (2002). En ella, hace referencia a un sistema de ideas sobre el cual se explica un suceso, estableciendo un vínculo forzado que prescinde de la relación causa-efecto. La imposición de esta ficción, no obstante, hace que, por el grado de afinidad que un público tiene con ese conjunto de ideas, esta quede materializada como la razón que justifica el suceso.
} 
locales. La planificación e implementación de políticas públicas y de estrategias corporativas obligan a la articulación de lo regional con lo transnacional, haciendo coexistir una estrategia de carácter universal con un espacio de ejecución reservado al ámbito doméstico del Estado-nación.

Saskia Sassen (2012) explica que "en tanto ciertas estructuraciones de lo global habitan lo que históricamente se ha concebido e institucionalizado como territorio nacional, esto genera la necesidad de una variedad de negociaciones" (p.33). Esta dinámica global, encarnada en lo local, requiere de estrategias de colaboración que regulen esta coexistencia. Aun en campos en lo que existe un consenso global acerca de los caminos que deberían tomar las políticas públicas, como seguridad, medioambiente, salud y educación, las estrategias deben planificarse de acuerdo con un nivel de ejecución que se da en escala local.

Además de los cambios ya descriptos sobre la idea de poder, se agrega la idea de su relocalización. El Estado-nación, como estructura, se inscribe en un nuevo modelo de diseño y toma de decisiones, en el que la concepción global afecta las condiciones sobre las cuales ejerce sus funciones.

Castells (2009) grafica la relocalización bajo estas premisas

Si las relaciones de poder existen en estructuras sociales concretas que se constituyen a partir de formaciones espaciotemporales ya no se sitúan primordialmente a nivel nacional, sino que son locales y globales al mismo tiempo, los límites de la sociedad cambian, lo mismo que el marco de referencia de las relaciones de poder que trascienden lo nacional (p. 43).

El poder constituye, ordena y demanda. Quitarle la potestad al Estado-nación obliga a revisar su rol y de qué manera queda inserto en un nuevo orden que lo considera un actor, pero no le otorga exclusividad. Sassen (2012) anticipa que los procesos globales actuales "pueden, y logran, desestabilizar la jerarquía de escalas centradas en el Estadonación” (p. 24). La naturaleza de este desafío queda manifiesta: en qué medida la institucionalidad clásica está en condiciones de compartir mecanismos de diseño y control de políticas, aceptando que su rol no ocupa el centro de la escena pública.

La política debe penetrar el tejido social desde una nueva acepción de poder y promover prácticas de participación y representación que descentralicen el rol de las instituciones. Si no lo logra, corre el riesgo de la disociación entre sus prácticas y las de la ciudadanía. La adaptación debe contemplar un tipo de institucionalidad que integre los mecanismos de circulación de información y asociación que conforman la idea de sociedad red.

El desafío que constituye para el Estado-nación aceptar esta coexistencia se expresa en la habilidad que desarrolle para involucrarse en esta nueva idea de poder. Castells (2009), en su prédica por la descentralización y el advenimiento de la era de la información, explica que "una forma especialmente relevante de dominación ha sido, a lo 
largo de la historia, el Estado en sus distintas manifestaciones" (p. 39). Es en la misma configuración del Estado en la que el autor encuentra esta capacidad de adaptación que se reclama para abordar el desafío de la coexistencia en la representación, entendiendo que son entidades históricas: "Por lo tanto, la cantidad de poder que ostentan depende de la estructura social general en la que operan" (p. 39).

La capacidad de adaptación de lo institucional a nuevas reglas de juego impuestas por el sistema global tiene menos dificultades que las que representa el desafío de articular a este sistema con el concepto de "cultura". Esto obedece a que la idea de institucionalidad ya alberga dentro de sí la suficiente carga de abstracción, erigida como una supraestructura más allá de las particularidades con las que se constituye y se organiza cada territorio.

La concepción de que lo institucional ofrece una representación cultural de las identidades que representa desconoce el grado de universalidad que está presente en la organización de los Estados y de sus funciones. La primera negociación de las prácticas culturales no es con los sistemas globales, sino con las instituciones que rigen los territorios en los que estas se desarrollan.

Esta institucionalidad contiene los elementos para lograr una coexistencia que le permita operativamente abordar el pasaje de lo local a lo global, ya que comparte algunos fundamentos expresados en lo universal. Su representación es la de un individuo tipo que no forma parte de ninguna cultura en particular. Jonathan Friedman (2001) justifica esta capacidad de adaptación de las instituciones en la inespecificidad identitaria que las constituye

\footnotetext{
La identidad cultural es algo que los individuos tienen y constituye la base de cierto tipo de identidad social, pero esta nunca es el contenido de las instituciones sociales de la sociedad. Estas últimas son tan abstractas y neutras como el individuo abstracto. Están constituidas por los roles asumidos por el individuo abstracto. La identidad cultural, por el contrario, es la particularidad concreta y no tiene ningún papel institucional en la sociedad moderna (Friedman, 2001, p. 58).
}

El marco de la coexistencia entre los sistemas globales y las prácticas locales abre un tipo de análisis que se puede abordar desde la etnología, considerándolos como espacios complementarios. Más allá de la particularidad de combinar perspectivas materiales y abstractas, la lógica de un espacio analizado y otro más amplio, cuya configuración incide en el primero, forma parte de la tradición de la antropología.

En referencia a esta coexistencia, Marc Augé (2008) manifiesta que siempre existen al menos dos espacios de análisis

\footnotetext{
el del lugar que estudia (un pueblo, una empresa) y otro lugar, más amplio, en el que aquel se inscribe y donde se ejercen influencias y presiones que no dejan de tener su efecto en el juego interno de las relaciones locales (p. 120).
} 
Que el sistema global no pueda ser definido como cultura no le impide ejercer un efecto condicionante sobre las prácticas locales.

Este espacio adquiere las características de un sistema, regido por sus prácticas y con base en la información. La idea de sociedad red se impone como un ámbito de pertenencia que permite su habitabilidad sin reclamar el abandono de la dimensión territorial. Para entrar en uno, no es necesario abandonar la otra. Se habitan redes y sitios, se intercambian likes y pantallas y estas prácticas acompañan, en simultáneo, los tránsitos materiales del territorio.

La ausencia de sentido representa una carencia en la prédica de los sistemas en red, en comparación con la idea de comunidad tradicional. No existe en ellos referencia cultural con base territorial que pueda prefijar objetivos que delimiten el alcance de las acciones ni su naturaleza. "En un sistema abierto no hay destino", sostiene Richard Sennett (2019, p. 252), definiendo que lo que se privilegia en estas estructuras no es el fin, sino el tránsito, por lo cual la garantía de funcionamiento está dada por el concepto de "libre circulación".

La relación entre lo global y lo local obliga a una reflexión sobre otro tipo de espacios, que exceden las características del territorio y, orientados a la idea de sistema, definen distintas prácticas que si bien no pueden ser definidas desde lo cultural, provocan una decisiva influencia en ellas. Si lo territorial se impone como límite y el poder se transforma en un medio, el desafío es "buscar las redes de poder socioespaciales (locales, nacionales, globales) que, en su intersección, configuran las sociedades" (Castells, 2009, p. 43).

Esto propone una nueva consideración acerca del espacio público, de la idea de sistema y entorno y de lo territorial, en el esfuerzo por definir mecanismos de colaboración y coexistencia entre lo global y lo local.

\section{Lo global y lo local en torno al espacio común}

La articulación entre lo local y lo global se define entre las dinámicas de colaboración y las tensiones que experimenta. El campo de definición de estas acciones impone condiciones y, a su vez, es modificado. En lo simbólico, el espacio público expresa un territorio que configura el sentido a partir de la relación entre política, medios y sociedad. El desplazamiento del ámbito local al global provoca cambios en su funcionamiento. La territorialidad, como representación de las relaciones y prácticas que se desarrollan en el ámbito local, permite un análisis acerca de las expresiones sociales que involucra. Cuando una identidad territorial encuentra dificultades para convertir sus problemáticas en insumo de la sociedad red, se produce un fenómeno de exclusión que, en nuestra región, puede encontrarse en las zonas periféricas de las grandes metrópolis. Cada uno de estos aspectos -espacio público, la territorialidad 
como expresión y la invisibilización de las prácticas sociales- se convierten en elementos de interpretación para repensar los mecanismos de articulación entre lo global y lo local.

\section{Reconfiguración del espacio público}

La concepción de un espacio público que centralice las actividades institucionales y comunicacionales, al otorgar sentido a la interacción entre sujetos, es un pilar de la sociedad moderna. Esta configuración permite explorar el grado de tensión en la dinámica de relación entre el subsistema mediático y el subsistema político. La premisa de que las acciones de estos dos conjuntos, en relación con sus funciones, tienen por objetivo conquistar el espacio común a través de la imposición de sentido, para condicionar la expresión de la sociedad civil, justifica una tradición de la opinión pública (Grossi, 2007).

Sobre este escenario, surge la idea de que el subsistema político, al cual queda reservada la actividad institucional, y el mediático, del que deriva la producción de medios masivos y digitales, disputan el sentido en un espacio de convergencia en el que también participa el subsistema civil, que agrupa las expresiones de la ciudadanía y las asociaciones vinculadas con el tercer sector. El resultado de esta interacción, en la que cada uno de los subsistemas pone en juego sus funciones proponiendo condiciones, es la opinión pública: el producto comunicacional del espacio público.

Sobre la base de estos subsistemas surgen las ideas de funciones, entornos y relaciones. Se define como "funciones" a los tipos de acciones que establecen vinculación entre elementos del mismo conjunto, como entornos al conjunto de elementos que están por fuera de cada sistema y a las relaciones como las acciones que vinculan al sistema y al entorno en función de las diferencias que los asisten (Luhmann, 2005).

La coexistencia y dinámicas de colaboración entre subsistemas en una escala local encuentra como campo común la territorialidad, que impone los límites al predominio de un conjunto por sobre el otro. La sociedad red desborda esta escala, ubicando a algunos subsistemas como mejor preparados que otros para adaptarse a la espacialidad global. Las funciones reservadas a lo corporativo mediático ${ }^{10}$ y las referentes a lo civil pueden inscribirse, adaptación mediante, en una lógica universal. Lo político, y su dimensión institucional, encuentran mayores dificultades de romper la noción de territorialidad, ya que su ámbito de influencia está determinado por los límites del Estado-

\footnotetext{
${ }_{10}$ El subsistema mediático ha adquirido complejidad en sus funciones. En su configuración dejan de ser exclusivamente medios de comunicación, para transformarse en parte de un holding de empresas más amplio y con actividades diversificadas. De esta manera, además de encontrar ventajas operativas en relación con el financiamiento, se incorpora también a un tipo de prácticas transnacionales que influencian en su posición respecto al sistema general, otorgándole ventaja respecto a otros que reservan sus actividades al ámbito local.
} 
nación y el ejercicio del poder no puede darse en ausencia del referente (Baudrillard, 2007).

Esta nueva configuración del espacio público, propuesta por la sociedad red, agrega un nuevo campo, en el que lo virtual hace las veces de territorio, reservando sus propias funciones y sus mecanismos de sistema y entorno. La sustitución de la representatividad en el ámbito común no inaugura su reflexión con la aparición de lo digital. Con la irrupción de los medios masivos, la política empieza a experimentar está sustitución en el desplazamiento, simbólico, que se da de la plaza a la pantalla. ${ }^{11}$

Este desplazamiento es parcial, con una alta carga simbólica. Si bien las discusiones que antes se materializaban, a escala territorial, en los espacios comunes, se mudan a los medios de comunicación; en lo operativo, plaza y pantalla coexisten. Esto origina reglas que permiten las relaciones en este hábitat. La sociedad de la información no reemplaza al espacio común propuesto por los medios masivos: plaza, pantalla y red conviven en distinta escala y con diferentes funciones.

Si reconocemos, en la simbología de la plaza, aquella conceptualización de lo público que se dirime en el ámbito parlamentario contando con una fuerte influencia de los grupos de presión de la sociedad civil; si ubicamos en la pantalla a una discusión política mediatizada en la que los intereses corporativos condicionan la discusión pública; la red, como tercer espacio, aparece como la imposición de la técnica al concepto en el que la multiplicación de los canales de expresión y el acceso a la información ubican a todas las acciones en el marco de lo público.

La coexistencia entre plaza, pantalla y red se encuentra condicionada por este tercer hábitat, ya que sus reglas de circulación y uso de la información imponen relaciones al entorno precedente. Las expresiones de escala territorial local, en la dimensión "plaza", y por un híbrido entre lo real y lo virtual, en lo que refiere a "pantalla", están condicionadas por la dinámica del espacio "red" en el que la frontera entre lo privado y lo público parece disolverse.

En el proceso de traducibilidad que opera la información -de analógico en digital y de bien de uso a bien de cambio- prolifera un tipo de contenido que disuelve la frontera entre la vida privada y la pública. Esta acción, expresada como regla, trasciende la dinámica de los individuos como usuarios y desborda hacia lo institucional; así, "el uso de los espacios sufre alteraciones en función de este procesamiento digital, que diluye la clásica oposición entre las esferas públicas y privadas" (Sibilia, 2013, p. 55).

\footnotetext{
11 La "plaza” como metáfora de espacio común nace de la tradición de lo público en Atenas, y su ágora, y se materializa en la construcción de un tipo de representatividad que encuentra en lo común el espacio de debate. A mediados del siglo XX, esta discusión empezó a darse en el escenario que proponían los medios masivos, que se convirtieron, así, en una plaza con soporte tecnológico. Esto deriva en una serie de reflexiones que referencia que la discusión pública se ha mudado de la plaza a la pantalla.
} 
El sistema global borra la frontera entre lo público y lo privado y recrea su propia lógica de sistema y entorno. Cada individuo, con auxilio de la técnica, puede considerarse un sistema respecto a los otros, lo que anula la dimensión colectiva. Las herramientas, garantes de sus funciones, son las que determinan su modo de vinculación con el conjunto externo. La multiplicación de la expresión individual, no inscripta en una lógica histórica ni territorial, anula la posibilidad de analizar al sujeto desde una evolución temporal. Esta multiplicación del presente cambia el concepto de espacio público, ya que este se convierte en "la expresión de múltiples actividades sincrónicas" (Sennett, 2019, p. 308).

Si las prácticas culturales, en escala territorial, aparecen como la posibilidad de interpretar relaciones sistemáticas entre sujetos en función de determinar conductas comunes (Geertz, 2005), la sociedad red propone otro tipo de análisis de relación en la que puede ser comprendida cada vez más como el resultado de "una coproducción entre hombres y máquinas" (Van Dijck, 2016, p. 60).

La virtualidad impone una serie de funciones que pueden ser inscriptas en un plano espacial. En ellas se experimenta una sustitución respecto a las prácticas culturales reservadas al territorio. Paula Sibilia (2013) caracteriza este fenómeno, explicando que

En el nuevo capitalismo de superproducción y marketing, afianzado más fuertemente en el consumo y en los flujos financieros que en la producción puramente industrial, saberes y poderes se entrelazan íntimamente con toda una serie de prácticas, discursos y placeres que refuerzan tanto su eficacia como su legitimidad sociopolítica. Sin embargo, también es cierto que toda una gama de tendencias asociadas al nuevo régimen (descentralización, privatización, virtualización, globalización) conspiran contra los viejos mecanismos de poder (p. 158).

Los mecanismos de circulación indican en la percepción del espacio instrucciones respecto a su uso, que tienen implicancias políticas. Si en la modernidad, imperio de la territorialidad, la idea de igualdad podía emparentarse con los mecanismos de participación política y representación en ámbitos institucionales, en la posmodernidad esta tradición de inter pares podría sustentarse en un tipo de socialización que, garantizada la idea del acceso a la información y conectividad, se expresa en la posibilidad de la libre circulación digital. ${ }^{12}$

Estas perspectivas se enfrentan con una jerarquización de usos, en el marco de la sociedad red, en la que algunas prácticas logran prevalecer por sobre otras. Van Dijck (2016), en referencia a una posible cultura de la conectividad, sostiene que "las tempranas expectativas de que la sociedad 2.0 diera lugar a una sociabilidad en plataformas

\footnotetext{
${ }^{12}$ Las lógicas de inclusión y exclusión permiten entender los desafíos de cada tiempo histórico en relación con su idea de igualdad. Las posibilidades de conectividad, el acceso a la información y el derecho a su libre circulación componen el conjunto de elementos que, en la idea de sociedad red, diferencian incluidos de excluidos y, sobre esa base, se erige una idea de garantizar las condiciones de igualdad.
} 
Santiago Aragón

La influencia del contexto como práctica comunicacional: el desafío de la integración en la sociedad de la información

signada por la equidad entre los usuarios y el acceso igualitario, resultaron utópicas" (p. 257).

Más allá de los desequilibrios de funcionamiento, originados por la disparidad de acceso y conectividad, el elemento que atenta contra la equidad es la falta de objetivos comunes que permitan establecer relaciones sistemáticas entre fenómenos diferentes, característica de la idea de comunidad. La expectativa de una cultura global que, con base en un espacio virtual, acredite una identidad común expresa un nuevo tipo de tensión en la cual "la sociabilidad, la creatividad y el conocimiento se trenzan en la trama del ecosistema, donde todas las actividades de codificación y explotación de la conectividad ocurren dentro de un mismo ámbito dominado por el espíritu corporativo" (Van Dijck, 2016, p. 268).

Considerando la prevalencia de estrategias comerciales y de identidad de marcas, las dinámicas sustentadas sobre lógicas de consumos, los sujetos en búsqueda de filiación ciudadana reconvertidos a usuarios, los sistemas cuyas funciones determinan elecciones que no pueden ser traducidas en valores y un espacio público sin ambición de colectividad, se impone un escenario en el que la sustitución de lo local por lo universal y de las prácticas culturales por los sistemas locales se torna improbable.

La imposibilidad de este desplazamiento, de vivencias producidas en el ámbito territorial a experiencias transitadas en la sociedad red puede expresar un nuevo tipo de conflictividad. Esta se expresa en la coexistencia de dos sistemas en los que la imposición de lo real y de lo virtual, como expresiones de cada uno de ellos, representa lógicas diferentes para abordar la idea de sistemas, funciones, entorno y relaciones.

Expresión de esta contrariedad representada por la imposibilidad de una parte de la ciudadanía de aceptar en un plano personal las reglas de juego impuestas por la sociedad red es el hecho de aferrarse a la modelización tradicional de espacios públicos y privados, entendiendo dentro de los primeros aquellos que contienen prácticas vinculadas con el apego a normas y valores que influyen en lo colectivo y se sujetan a lo institucional, dejando reservado para lo segundo aquellas que forman parte de lo doméstico, lo familiar y lo que sucede intramuros.

Van Dijk (2016) lo ejemplifica en la anécdota individual de un sujeto que empieza a resultar sospechoso por negarse a compartir sus vivencias en las redes sociales

\footnotetext{
Muchos valores que Pete alguna vez había dado por sentados -el de privacidad, el de un espacio público libre de los intereses comerciales, el derecho a saber quién controla determinado espacio social- ya no resultaban tan importantes para sus pares. Era él quien parecía ser la excepción, quien contrariaba la norma (p. 279).
}

Sobre esta ambición de sustitución que, en el marco de la "era de la información", experimenta lo virtual sobre lo territorial surgen dos aspectos a considerar. El primero se define en el interrogante acerca del destino de los espacios físicos ante la imposición 
de un modelo de sociedad red que, considerado como sistema, se ubica como entorno y se vincula a partir de la diferencia con toda expresión que no responda a las funciones que lo constituyen. El segundo es si la dimensión territorial, continente de las prácticas culturales, puede resistir la presión que sobre sus expresiones tiene la variable universal. Asumiendo la respuesta negativa, producto de la asimetría evidente entre estas prácticas y el sistema global, surge el interrogante de cómo el territorio, como elemento preexistente, puede articular expresiones que se resistan a esta ambición de sustitución, logrando imponer algunas características que, desde la cultura, inscriban prácticas locales en sistemas universales.

\section{El territorio como expresión social}

La noción de "espacialidad" provista por la sociedad red provee al sujeto de una lógica de "píldora azul"13 que le permite garantizar el tránsito sin reparar en las consecuencias que, en la personal, guarda esta decisión. En la continuación de esta metáfora, la red surge como una infinita combinación de itinerarios individuales, dotados del don de la autoconfiguración. El espacio físico tradicional cae en desuso y representa lo pasado.

Existe una territorialidad que no logra inscribir sus prácticas en el marco ampliado del escenario global, víctima de un doble cierre de exclusión. Esta imposibilidad de adaptación está expresada en disvalores.

El primer cierre se da en la dicotomía de lo global-local, ubicando dentro del primer término a aquellos elementos que están diseñados bajos los parámetros de los sistemas universales. En ellos, el centro de su actividad reside en su capacidad para la circulación de información, ya sea como parte de una nueva institucionalidad de carácter transnacional, ${ }^{14}$ como nodo del capitalismo financiero global o como experimento de nuevas formas de socialización en red y tiempo real. Dentro de lo local, se hallan las prácticas culturales arraigadas a las coordenadas espacio-temporales definidas, una apuesta a la territorialidad como condición de identidad y la apelación a la variable histórica como explicación de los sucesos del presente.

El segundo cierre de exclusión se da en los términos clásicos de centro y periferia, aunque con dos variantes respecto a este enfoque. En primer término, la apelación ya no refiere a dos materialidades, sino a una espacialidad y a una territorialidad, entendidas

\footnotetext{
${ }^{13}$ La metáfora de la "píldora azul", originada en la saga de las películas Matrix, forma parte de la cultura popular. En la película, a Neo, el protagonista, le son ofrecidas una píldora azul y otra roja. Su decisión deriva en dos modos de vida. Si elige la azul, puede olvidar todo lo sucedido y transitar la realidad virtual, mientras que con la roja, se libera de la matrix y vuelve a la vida real.

${ }^{14}$ Lo transnacional aparece como condición precedente e implica una filosofía que, con base en la colaboración económica, reconfigura las prácticas que se dan en el marco de un Estado-nación, en lógicas colaborativas que construyen un nuevo espacio común
} 
como el enfrentamiento entre sistemas generales y prácticas particulares; esto condiciona el segundo elemento: a diferencia de los enfoques desarrollistas en los que lo periférico encarna un estadio primario y precedente, plausible de ser convertido en centro a partir de la aceptación de determinadas reglas y funciones, el entorno evidencia síntomas de exclusión permanente. Lo que no se ha integrado no lo hará, porque es la condición para que el centro siga operando bajo funciones de carácter universal.

No toda definición expresada en los términos de referencia de este doble cierre de exclusión puede ser abordada como la dicotomía entre una espacialidad abstracta, definida como sistema, y una localización concreta, indicada como territorio. Hay una expresión material de la espacialidad que recrea, en sus prácticas, la universalidad demandada por la idea de sociedad red: las ciudades globales.

El concepto de ciudad global es expuesto por Saskia Sassen en su obra Una sociología de la globalización (2012)

\begin{abstract}
La investigación sobre las ciudades globales se centra en los territorios estratégicos de la economía global. Las ciudades globales son territorios subnacionales donde se entrecruzan múltiples circuitos globales, lo que las ubica en varias geografías transfronterizas, cada una constituida en términos de ciertas prácticas y de actores específicos y con un alcance propio. Presenta un panorama de la globalización que difiere de las descripciones centradas en los mercados globales, el comercio internacional o las respectivas instituciones supranacionales (p. 32).
\end{abstract}

Esta expresión de territorialidad adaptada basa su supervivencia en la posibilidad de transpolar sus prácticas sociales, inscribiéndolas en un modelo económico que las adopta bajo un rol definido. Así, condiciones logísticas, geolocalizaciones estratégicas y sociedades abiertas aparecen como privilegiadas para el desplazamiento que implica su consideración como "ciudad global".

En este tránsito, "acumulan concentraciones inmensas de poder económico, mientras que otras ciudades que en su momento fueron centros industriales hoy sufren una decadencia desproporcionada" (Sassen, 2012, p. 143). Esto no puede ser considerado como una tercera variable en torno a la idea de cierre de exclusión ya referida, sino como consecuencia del doble cierre descripto.

La consideración de que el espacio es una producción social (Lefevbre, 2013) expresa el alto grado de particularidad por el cual un territorio puede ser considerado un lugar antropológico. ${ }^{15}$ En esta tradición se inscriben los análisis orientados a expresar que las ciudades son reflejo del tipo de prácticas que se desarrollan en su interior. Los modos de relación, de intercambio, lo permitido y lo prohibido aparecen bajo la forma

\footnotetext{
15 En la definición de Marc Augé (2008), el "lugar antropológico" corresponde a una espacialidad en el que se han cargado características identitarias, relacionales e históricas, que la convierten en una territorialidad que expresa el tipo de relaciones que se dan en torno a ella.
} 
de edificios, arterias, cruces y centros. La territorialidad entendida como humanidad materializada.

La tradición del urbanismo moderno ubica en la configuración física de las ciudades la recreación de las funciones fisiológicas, de manera que la habitabilidad y el tránsito del cuerpo puede ser pensado como metáfora del territorio y viceversa. Sennett (2019) ${ }^{16}$ profundiza este concepto al referir que "las medidas a escala humana en un medio construido parecerían basarse, lógicamente, en el tamaño del cuerpo humano” (p. 241).

El grado de individualización expuesto en las prácticas comunicacionales -incluyendo la disolución de la frontera entre lo público y lo privado- y el cese de las mediaciones institucionales y culturales, que permiten incorporarse en un sistema global sin más trámite que el acceso a la conectividad, expresan un tipo de diversidad aceptada. La fragmentación de la experiencia personal y la multiplicidad de identidades puestas en juego se redimen a través del tránsito propuesto por la sociedad red. Las condiciones de circulación logran instalar la condición de adaptabilidad que presupone una diversidad homogeneizada.

En contraposición, las distintas variantes de territorialidad que no superan el doble cierre de exclusión, por su carácter local o periférico, incluyendo las prácticas culturales que se desarrollan en torno a estos espacios, forman parte de una realidad invisibilizada. Lo excluido no adquiere forma, no habita ni circula, esta diversidad no homogeneizada aparece como el aspecto residual de la sociedad red.

Lo global se ampara en un tipo de relaciones que legitima la exclusión bajo argumentos técnicos, prescindiendo de territorialidades que por sus características no pueden ser incorporadas al sistema. Se expresa una antinomia en torno a la idea de lugares que confronta un tipo de espacio ideal, expresado por la potencialidad y sin representación de las carencias, con otros en los que, conjuntamente al lugar que no logra ser integrado, envejecen los tipos de vinculación que forman parte de su estructura.

Estas territorialidades, y sus prácticas, son consideradas como efectos colaterales; y sus síntomas, tratados como contraindicaciones de lo deseable en relación con el sistema global y la sociedad red. Lo que se conceptualiza como excluido se operativiza como negado. La invisibilización representa la condena del sistema a su entorno. Representa una fase inicial en la lógica centro-periferia, pero se extiende a los modos de relación entre las ciudades globales y las que, en el marco de un mismo Estado, no adquieren esta categoría. Lo poblacional excluido tiende a transformarse en entorno a sus propios centros territoriales y su expresión como comunidad se torna amenazante. La dinámica de la invisibilización evidencia que la manifestación de la carencia es, para el sistema, imputable.

\footnotetext{
${ }^{16}$ En Carne y piedra (Sennett, 1997) se realiza una evolución del concepto de "ciudad", ejemplificando como, a lo largo de la historia, se han ido agregando funciones al tránsito territorial conforme se generaban nuevos modos de relación entre los sujetos.
} 
Lo periférico, en términos de hábitat, deriva en la idea de expulsiones (Sassen, 2015). Pueden considerarse interterritoriales cuando son regiones enteras las que padecen los efectos de la exclusión, o intraterritoriales cuando la distinción se efectúa al interior de las propias comunidades. El interrogante se desplaza hacia las condiciones que derivan en esta invisibilización, entendida como una imputación en torno a las prácticas que, en relación con lo cultural, adopta lo periférico. María Carman (2011) resume el interrogante en “ ¿ cuáles son las representaciones de impureza que subyacen a las prácticas de separación física de los sectores populares y su expulsión de la ciudad?" (p. 257).

\section{Prácticas y exclusión en el conurbano bonaerense}

La exclusión expresa algo más que la dificultad de incorporar las prácticas sociales en el sistema vigente. Tiene consecuencias sobre el modo de vida, las posibilidades, las oportunidades y se expresa en lógicas de marginación con consecuencias en lo social. Sobre este resultado, además, se produce un efecto priming, cuya atribución tiene derivaciones estéticas que profundizan las consecuencias de la marginación: en el análisis de lo territorial, se asocia "a los pobres con el caos, el desorden y la contaminación, presumiendo que ellos habitan un universo puramente físico, centrado en la actividad práctica y el interés utilitario" (Carman, 2011, p. 252).

Esta definición, que agrupa dinámicas de exclusión, condiciones de marginación, consecuencias de pobreza, invisibilización de las prácticas y desplazamiento en lo territorial, está expuesta en los escenarios que representan los conglomerados urbanos que se construyen en torno a las grandes ciudades de nuestra región. Así, Buenos Aires, San Pablo, Lima o Guayaquil expresan esta versión a escala de centro y periferia que se da entre las ciudades globales y sus entornos territoriales.

A modo de ejemplo, una breve conceptualización sobre el Gran Buenos Aires, puede ofrecernos reflexiones sobre cómo opera la dinámica de invisibilización y exclusión y cuáles son sus consecuencias. El conurbano bonaerense es materia de análisis social, económico y político por el peso de sus variables estadísticas y su influencia en la realidad argentina. El 35\% de los habitantes del país residen en este territorio; la brecha de desigualdad que se expresa en su interior impone una conflictividad que se traslada más allá de sus límites y el pulso de lo que sucede en lo político y social se da en este hábitat. Sobre esta caracterización aparece la problemática: la imposibilidad de legitimar sus prácticas inscribiéndolas en un sistema que trascienda sus propios límites territoriales, para incorporarse a la cultura.

Adrián Gorelik sintetiza esta dificultad en la idea de que "el Gran Buenos Aires, el fenómeno urbano de mayor envergadura entre los años cuarenta y los setenta, que 
alojó todo el crecimiento de la metrópoli más importante del país, no fue un objeto diferenciado de representación" (Gorelik, 2015, p. 55).

En el análisis de la producción literaria sobre el conurbano bonaerense y las prácticas que se dan en su interior predomina un tipo de estudios que privilegian la reflexión sobre los sectores más favorecidos y desfavorecidos de este territorio. Así, las investigaciones sobre el modo de vida en los barrios de emergencia y las villas miseria encuentra, en espejo, un volumen similar para analizar qué es lo que sucede dentro de los barrios cerrados y los countries. Lo llamativo es que estos dos sectores representan, en conjunto, alrededor del $20 \%$ de los 13 millones de habitantes que se estima residen en el conurbano bonaerense. ${ }^{17}$ Sobre las prácticas, los modos de relación y los deseos aspiracionales de alrededor de 10 millones de personas, prácticamente no hay literatura producida, como si el cerrojo de la invisibilidad, atribuido al entorno, desconociera la potencia del volumen y la jerarquía de las proporciones. Gorelik (2015) explica que de su estudio sobre este territorio aparece "la casi completa ausencia de referencias al Gran Buenos Aires en la literatura y el cine del siglo XX" (p. 51).

A la idea de conurbano la precede una noble negación: la imposibilidad de ser nombrado y la de ser habitado. Sobre la primera se menciona la particularidad de no poder contar con un gentilicio que haga referencia a la territorialidad. Ni completamente bonaerense ni evidentemente porteño, ${ }^{18}$ la definición es un híbrido explicado por la negación derivada de la diferenciación. La imposibilidad del hábitat corresponde a una problemática más compleja que lo anecdótico del gentilicio: la inseguridad de territorios que expresan un evidente contraste en términos de ingresos y posibilidades entre los sectores de mayor y menor poder adquisitivo, con consecuencias concretas sobre las condiciones de habitabilidad, tránsito y uso de los espacios comunes por parte de los sectores medios.

Sobre esta definición, el Gran Buenos Aires aparece como la representación de lo no deseable. Rodrigo Zarazaga, en su obra Conurbano infinito (2017), expone las conclusiones que derivan de su estudio.

A menudo el Conurbano se presenta al ciudadano de la CABA, pero también al del resto de la Argentina, como "un país otro", un lugar distinto donde acecha la amenaza. Para muchos, es un mero amalgamiento de pobres, sobrevivientes y redes ilegales. Para un imaginario, es el país imposible de rescatar (p. 9).

\footnotetext{
${ }_{7}$ Las estadísticas surgen de distintos organismos públicos, que tienen como objeto de estudio al conurbano bonaerense, utilizando como base el Censo poblacional de 2010

18 Esta característica, puesta en el plano de curiosidad, tiene que ver con la antítesis que se produce entre "lo porteño" y "lo federal" en representación de la Ciudad de Buenos Aires, los primeros, y del resto del país, los segundos. Quien habita el conurbano bonaerense no es considerado porteño por estos, pero sí es "imputado" de esta condición por quienes se reivindican federales. En tanto, los habitantes del conurbano tampoco pueden ser definidos como bonaerenses, ya que esta apelación suele utilizarse para quienes habitan en el interior de la provincia de Buenos Aires. Permanecen así en la imposibilidad de ser nombrados más allá de la negación de otros gentilicios.
} 
Esta imposibilidad de ser considerado más que como entorno tiene consecuencias sobre lo institucional. La representación de un Estado que, en su estructura, adquiere el mismo grado de informalidad y contingencia que el territorio que administra, con la dificultad evidente de instalar categorías en la que lo vivencial pueda ser considerado como algo más que accidental. La referencia a un Estado Gólem ${ }^{19}$ (Zarazaga, 2017) es la metáfora que mejor resume las características de lo institucional en su ambición de gestionar lo que en términos de sistema considera periférico.

En el análisis, surge como evidencia que lo institucional tiene menos dificultades de interacción para establecer dinámicas de adaptación a lo global, de las que experimenta para gestionar la complejidad expresada en sus propios territorios. Esto es el producto de la diversidad no homogenizada que, caracterizada como periférica, queda por fuera del sistema. Para este sector no hay previsión de funciones comunes ni estrategias de incorporación de sus prácticas al conjunto.

Las condiciones de lo periférico territorial han mutado. En la referencia original representaba la experimentación de lo novedoso, de lo que aún no había conquistado el centro. Esto le confería una estética vinculada con lo dinámico y lo disruptivo, albergando tendencias urbanas alternativas que luego se convertirían en hegemónicas. En la actualidad, representa lo marginal, lo no deseable, lo que queda considerado como ajeno al sistema.

Gorelik (2015) realiza esta distinción caracterizando los dos ciclos mencionados

$$
\begin{aligned}
& \text { Si en el primer momento expansivo, la periferia (todavía dentro de la capital) funcio- } \\
& \text { nó como un territorio de experimentación urbana, social y cultural que transformó el } \\
& \text { conjunto de la ciudad, en un segundo momento, en cambio, la periferia (el gran Buenos } \\
& \text { Aires) tendría apenas el papel de territorio de reproducción -en condiciones cada vez más } \\
& \text { degradadas- de la jerarquía urbana, social y cultural ya consolidadas (p. 43). }
\end{aligned}
$$

Más allá del análisis que se desprende de los dos momentos mencionados, queda expresada la certeza de que la relación entre periferia y centro puede conferirle a este un dinamismo propio de lo emergente. ¿La condición?, visibilizarlo como algo más que entorno, estableciendo prácticas de interacción que permitan incorporar su influencia en el sistema: transformar el entorno, como figura pasiva, en contexto, como estructura activa.

En relación con la problemática territorial que evidencia el conurbano bonaerense, Gorelik (2015) propone, en forma de interrogantes, algunos caminos para explorar

\footnotetext{
${ }^{19}$ La figura del Gólem, proveniente de la mitología hebrea, aparece como la representación de un ser que, dotado de vitalidad por el hombre y surgido de la materia inanimada, mantiene un comportamiento autómata, precisando de instrucciones para poder funcionar, aunque, en determinadas circunstancias, adquiere características propias pudiendo incluso rebelarse contra quienes lo crearon.
} 
esta interacción y dotarla de sentido, que pueden trasladarse al desafío de contextualizar el entorno

\begin{abstract}
¿Cómo pensar sin reduccionismos las relaciones entre esta inédita producción cultural del Conurbano y las nuevas lógicas sociourbanas que nos muestra la realidad metropolitana? ¿Cómo una reparación simbólica ante las pérdidas por la desintegración de la ciudad expansiva? ¿Cómo el último estertor de unas clases medias cercadas por la polarización social? ¿Cómo una búsqueda comunitarista por definir dentro del nuevo mapa fracturado otras identidades que doblan la apuesta de la fragmentación, volviéndola positiva? ¿O tal vez como el intento de traducir las nuevas identidades de grupo en una reorganización general de la cultura y la sociedad? (p. 65).
\end{abstract}

El desafío de visibilizar lo periférico, otorgándole el carácter de contextual, representa materializar la potencialidad a través de una dinámica de intercambio virtuoso. Aceptar las características que expresa el contexto, con la visibilización y la incorporación de sus prácticas, revierte la negación de las identidades de los habitantes de estos territorios, ya que la dinámica de intercambio vuelve cierta la afirmación de que "la identidad está determinada por la posición que se ocupa en una red más amplia de relaciones" (Friedman, 2001, p. 67).

\title{
Conclusión: la emergencia del contexto
}

A modo de conclusión, surgen desafíos que, en materia de articulación, pueden permitir explorar dinámicas de coexistencia entre prácticas culturales y sistemas globales.

El espacio público, más allá de sus funciones como sistema, surge como un ámbito de convergencia entre lo institucional y lo territorial, donde la complejidad de las expresiones sociales puede incorporar sus problemáticas y particularidades a los contenidos que aparecen expresados en la agenda mediática y a los objetivos de planificación de las políticas públicas.

Reivindicar la potencialidad de lo contextual permite operacionalizar, a través de la comunicación, los mecanismos para revertir la inestabilidad y la amenaza que se le atribuyen al entorno, derivadas en prácticas que, en ocasiones, afecta a sociedades enteras. Su visibilización asume el desafío de la integración como mecanismo para revertir el doble cierre de exclusión, caracterizado por la condición local y periférica, y permite asumir la dificultad que experimenta la territorialidad en la adaptación a lo universal.

La coexistencia de sistemas globales y prácticas culturales debe darse bajo condiciones que permitan abordar la diversidad de aquellos elementos que no pueden ser homogeneizados por los sistemas de información en red. La reorganización del territorio, integrando lo periférico a lo central, surge mediante la constitución de espacios 
comunes que estén en condiciones de inscribir las manifestaciones individuales en prácticas colectivas. Conviene, para esta tarea, revitalizar los alcances del lugar antropológico y de sus características identificatorias, relacionales e históricas (Augé, 2008).

La posibilidad de coexistencia de espacios públicos y privados, y de los sistemas y territorios, permite habitar una geografía local y una red al mismo tiempo sin que esto represente para el sujeto una imposibilidad: las condiciones de circulación lo permiten. El desafío de la doble habitabilidad puede resumirse en un dilema: ¿las prácticas y valores que uno de los espacios propone son aplicables a las vivencias que se registran en el otro?

La capacidad de romper el estigma de lo periférico, constituyendo la espacialidad en el lugar a través de lo identificatorio, consiste en reconocer como cultural el proceso por el cual un sujeto puede explicar su propia individualidad a partir de la referencia a los modos de relación del hábitat donde se desarrolla. Respecto a lo relacional, esta característica se extiende al tipo de rol y de funciones que un individuo adquiere en el marco de su sistema territorial y que solo puede entenderse en un conjunto y bajo coordenadas espaciotemporales específicas. Lo histórico pretende recuperar para el territorio la condición de inscribir el presente en un devenir. Así, las relaciones de casualidad pueden ser explicadas por condiciones precedentes, y los sucesos que han configurado las características actuales de un territorio pueden ser invocados como razones en este puente con el pasado.

Lo identificatorio, lo relacional y lo histórico aparecen como las operaciones mediante las cuales el espacio se reconvierte en lugar antropológico. Los tres mecanismos expresan la influencia de la cultura en la vinculación entre sujeto y territorialidad.

Contextualizar es considerar al espacio como una expresión de las vivencias y las relaciones que en él se desarrollan. El espacio, sin las prácticas que en él se inscriben, solo puede ser pensado desde una naturaleza potencial: "Si los seres humanos están ahí, están en principio en espacios que se han abierto para ellos porque ellos les han dado forma, contenido, extensión y duración relativa al habitarlos" (Sloterdijk, 2006, p. 52).

Los mecanismos de representación en el marco de la sociedad red deben ser abordados desde la emergencia comunicacional. Esta decisión permite explorar alternativas para construir una mirada que profundice los rasgos de la diversidad. El sesgo de reducir las prácticas a un mecanismo informacional que privilegia las condiciones de circulación limita la participación a la capacidad de adaptación técnica al nuevo escenario.

A la homogeneización de ese modo de ver las cosas, la comunicación responde con la heterogeneización dada por la polivalencia de lo cotidiano, por la diferenciación étnica-simbólica, por la pluralidad espacios-tiempos de interacción afectiva o comunitaria. El concepto de comunicación interesa, de esta manera, como el de una práctica de apertura del ser-en-el-mundo (Munis Sodré, 1998, p. 101). 
La comunicación produce las operaciones de sentido por las cuales las características complejas que aparecen en torno a la potencialidad de lo diverso se transforman en las identidades que definen el alcance de las relaciones en un territorio determinado. Cada elemento debe ser entendido como parte de un contexto que condiciona la naturaleza del sistema con el que se vincula.

Por esto, la operación por la cual la uniformidad periférica se transforma en diversidad activa es comunicacional. Sus mecanismos permiten transformar al entorno en contexto, a través de operaciones de sentido que transforman las funciones de los distintos elementos en relaciones de un sistema común.

En este proceso, el contexto pasa a ubicarse dentro del espacio común mediante las prácticas culturales y es constitutivo de él. La reflexión sobre su influencia debe surgir de su articulación con el sistema en el que opera y se inscribe. Complejizar la reflexión sobre su composición deriva en la caracterización de sus variantes territorial, poblacional o situacional.

El contexto territorial es aquel que, por las modelos de organización de una comunidad, expone formas de vinculación distintas del sistema con el que interactúa, condicionando las prácticas que se dan en este. Dentro de esta variante, las formas de relación suelen ser más informales, los medios de expresión exploran canales alternativos y las dinámicas de vinculación prescinden de los mecanismos institucionales.

El contexto poblacional ubica en las identidades de un conjunto los elementos de distinción respecto al sistema considerado centro. La diversidad de estas características puede ser pensada como un conjunto único, como varios subconjuntos o abordarse desde la entera complejidad. Este contexto poblacional no necesariamente se ubica como circundante de lo central, sino que aparece integrado a él en múltiples nodos de intervención e interacción.

El contexto situacional está definido por acontecimientos que, por sus características anómalas y su relevancia extrema, provocan hechos que se distinguen de las prácticas habituales de una sociedad. Esta situación, que desborda el margen crítico, tiene consecuencias sobre el sistema e implica la necesidad de adoptar estrategias diferentes para la adaptación y la supervivencia de este.

Cuando lo territorial, lo poblacional y lo situacional son abordados bajo la inespecificidad de lo periférico, las consecuencias de esta generalización tienen impacto en las dinámicas de convivencia. La imposibilidad de definir los rasgos sobre los cuales se estructura esta diferenciación lleva a considerarlas como disfunciones, obligando al sistema a un nivel de abstracción, y de clausura, que limita las prácticas culturales que se desarrollan dentro de él.

Si estas variables se articulan en torno a una estrategia comunicacional que las convierte en contexto, el sistema explora dinámicas de colaboración con lo emergente, 
Santiago Aragón

La influencia del contexto como práctica comunicacional: el desafío de la integración en la sociedad de la información

incorpora prácticas para gestionar lo diverso y propone mecanismos para enfrentar las crisis. La coexistencia entre lo territorial y lo espacial, y las prácticas y los sistemas, surgen así de la capacidad comunicacional de transformar la información del entorno en relaciones de integración.

Lo identitario y lo cultural no están definidos exclusivamente por los rasgos constitutivos: también pueden ser entendidos como los mecanismos de relación que se exploran, los modos que se eligen para gestionar la diferencia y el grado de apertura de las prácticas de relacionamiento. La identidad es una forma de estar en el mundo.

Atribuirle valor al contexto abre dos posibilidades: articula sistemas de relación entre sistemas y territorios donde los modos de vinculación de los primeros se ajusten a la naturaleza de las funciones que se expresan en los segundos, o incorporar a las prácticas culturales la potencialidad, la diversidad y el dinamismo propio de los espacios emergentes sin considerarlos una amenaza a la naturaleza del sistema.

La prédica de poner en valor lo que no ocupa el centro de la escena expresa una reivindicación sobre la importancia de planificar una gestión con eje en la diversidad. Advertir acerca de la imposibilidad de considerar a los mecanismos de acceso a la información y condiciones de circulación como prácticas culturales no significa desconocer el aporte que, en materia de visibilización, control y formas de vinculación pueden ofrecer las nuevas tecnologías.

El desafío de la integración entre lo global y lo local, expresados en sistemas y prácticas, encuentra en el contexto un elemento para el abordaje de lo que se considera distinto. Se podría explorar una dinámica colaborativa entre espacios y territorios si la cultura estuviera en condiciones de proponer mecanismos que visibilicen las relaciones similares entre elementos diferentes mediante estrategias colaborativas. El contexto es ese conjunto de operaciones sobre las cuales lo diverso logra influencia, garantizando su inclusión en el sistema y promoviendo la integración.

\section{Referencias}

Augé, M. (2008). Los no lugares: espacios del anonimato. Barcelona: Gedisa.

Baudrillard, J. (2007). Cultura y simulacro. Barcelona: Kairós.

Carman, M. (2011). Las trampas de la naturaleza. Buenos Aires: Fondo de Cultura Económica.

Castells, M. (2009). Comunicación y poder. Madrid: Alianza Editorial.

Contissa, N. (2000). La dictadura del sí mismo (no publicado).

Dufour, D. (2007). El arte de reducir cabezas. Buenos Aires: Paidós.

Eagleton, T. (2001). La idea de cultura. Barcelona: Paidós.

Friedman, J. (2001). Identidad cultural y proceso global. Buenos Aires: Amorrortu.

Geertz, C. (2005). La interpretación de las culturas. Barcelona: Gedisa. 
Austral Cmunicación

Volumen 9, número 2 (Diciembre de 2020): 187-215. ISSN 2313-9129

Gorelik, A. (2015). Terra incógnita: para una comprensión del Gran Buenos Aires como Gran Buenos Aires. En Kessler, G. (dir.). Historia de la provincia de Buenos Aires: el Gran Buenos Aires (pp. 21-69). Buenos Aires: Universidad Pedagógica Nacional. Grossi, G. (2007). La opinión pública: teoría del campo demoscópico. Madrid: CIS. Harvey, D. (2004). La condición de la posmodernidad. Buenos Aires: Amorrortu. Lefevbre, H. (2013). La producción del espacio. Madrid: Capitán Swing. Luhmann, N. (2005). El arte de la sociedad. México: Herder. Lyotard, J. (1997). La condición postmoderna. Madrid: Cátedra. Mouffe, C. (2007). En torno a lo político. Buenos Aires: Fondo de Cultura Económica. Ramonet, I. (2003). La tiranía de la comunicación. Madrid: Debate.

Sassen, S. (2012). Una sociología de la globalización. Buenos Aires: Katz.

Sassen, S. (2015). Expulsiones: Brutalidad y complejidad en la economía global. Buenos Aires: Katz.

Sennett, R. (1997). Carne y piedra. Madrid: Alianza Editorial. Sennett, R. (2019). Construir y habitar. Barcelona: Anagrama. Shumway, N. (2002). La invención de la Argentina. Buenos Aires: Emecé. Sibilia, P. (2013). El hombre postorgánico. Buenos Aires: Fondo de Cultura Económica. Sloterdijk, P. (2006). Esferas III. Madrid: Siruela.

Sodré, M. (1998). Reinventando la cultura. Barcelona: Gedisa. Sztompka, P. (2012). Sociología del cambio global. Madrid: Alianza Editorial. Van Dijck, J. (2016). La cultura de la conectividad. Buenos Aires: Siglo XXI. Zarazaga, R. (2017). Conurbano infinito. Buenos Aires: Siglo XXI. 